\title{
Prevention and Treatment of Periodontal Diseases in Primary Care
}

\author{
Abstracted from \\ Prevention and Treatment of Periodontal Diseases in Primary Care, \\ Dental guidance. Scottish Dental Clinical Effectiveness Programme, Dundee, June 2014. \\ ISBN 9781905829170 \\ Address for correspondance: Scottish Dental Clinical Effectiveness Programme, \\ Dundee Dental Education Centre, Frankland Building, Small's Wynd, \\ Dundee DD1 4HN, Scotland. E-mail: scottishdental.cep@nes.scot.nhs.uk
}

Scope and purpose The aim of this guidance is to support the dental team to; manage patients with periodontal diseases in primary care appropriately; improve the quality of decision making for referral to secondary care; improve the overall oral health of the population. It focuses on the prevention and non-surgical treatment of periodontal diseases and implant diseases in primary care. The surgical treatment of periodontal and implant diseases and the management of patients by periodontal specialists or in a secondary care setting are outwith the scope of this guidance and are not discussed in detail. The guidance is based on existing guidelines, including those from the British Society of Periodontology, relevant systematic reviews, research evidence and the opinion of experts and experienced practitioners.

Methodology The methodological approach is based on the international standards set out by the Appraisal of Guidelines Research and Evaluation (AGREE) Collaboration (www.agreetrust.org). The guiding principle for developing guidance within SDCEP is to first source existing guidelines, policy documents, legislation or other recommendations. Similarly, relevant systematic reviews are also initially identified. These documents are appraised for their quality of development, evidence base and applicability to the remit of the guidance under development. In the absence of these documents or when supplementary information is required, other published literature and unpublished work may be sought.

Review and updating The guidance will be reviewed in three years and updated accordingly.

Recommendations Recommendations are provided for assessment and diagnosis; changing patient behaviour; treatment of gingival conditions; periodontal conditions; long term maintenance; management of patients with dental implants; referral and record keeping. The key recommendations highlighted are:

- Assess and explain risk factors for periodontal diseases to patients

- Screen all patients for periodontal diseases at every routine examination

- Carry out a full periodontal examination for patients with BPE scores 3,4 and *

- Use the Oral Hygiene TIPPS (talk, instruct, practise, plan, support) behaviour change strategy to address inadequate plaque removal

- Raise the issue of smoking cessation where appropriate

- Encourage patients to modify other lifestyle factors that may impact on their oral health

- Ensure the patient is able to perform optimal plaque removal

- Remove supra-gingival plaque, calculus and stain and sub-gingival deposits
- Ensure that local plaque retentive factors are corrected

- Remove supra-gingival plaque, calculus and stain and correct any local plaque retentive factors. Carry out root surface instrumentation at sites $\geq 4 \mathrm{~mm}$ probing depth where sub-gingival deposits are present or which bleed on probing

- Do not use antimicrobial medication to treat chronic periodontitis

- Remove supra-gingival plaque, calculus and stain and sub-gingival deposits and ensure that local plaque retentive factors are corrected

- Assign an individual's risk level based on the patient's medical history and oral health status and schedule recall appointments accordingly

- Ensure the patient is able to perform optimal plaque removal around the dental implant(s)

- Examine the peri-implant tissues for signs of inflammation and bleeding on probing and/or suppuration and remove supra- and submucosal plaque and calculus deposits and excess residual cement

- Perform radiographic examination only where clinically indicated

- Consult any locally produced referral guidelines and the BSP 'Referral Policy and Parameters of Care' to determine if the patient is a suitable candidate for referral

- Carry out initial therapy to address inadequate plaque removal, smoking status (if applicable) and to remove supra- and sub-gingival deposits

- Provide supportive periodontal therapy and monitoring for patients who have been discharged from secondary care

- Record the results of the periodontal examinations (basic and/or full) carried out and the current standard of oral hygiene

- Record the diagnosis, suggested treatment plan and details of costs

- Document any discussions you have with the patient, for example, treatment options, risks and benefits of treatment, oral hygiene advice, smoking cessation, alcohol consumption and/or other lifestyle factors.

Research recommendations There is a need for high-quality research carried out within an appropriate governance framework to improve the evidence base in the following areas:

- barriers and facilitators to the delivery of oral hygiene interventions in primary care;

- behaviour change interventions to improve inadequate oral hygiene;

- optimal timescales for provision of routine supra-gingival debridement (dental prophylaxis) and supportive periodontal therapy;

- effectiveness of supportive periodontal therapy regimens;

- effectiveness of supportive therapy regimens to maintain peri-implant tissues;

- effectiveness of interventions to treat peri-implant mucositis and peri-implantitis. 
Consensus is urgently required on the importance and validity of surrogate periodontal outcomes (eg bleeding on probing, changes in clinical probing depth and clinical attachment level and bone levels) and their relationship to true outcomes (eg tooth loss and patientcentred outcomes) so that consistency can be achieved across studies. There is also a need for independent research into the effectiveness of oral hygiene tools such as toothbrushes, interdental aids, toothpastes and mouthwashes and gels containing antibacterial agents.

\section{Commentary}

Clinical practice guidelines ('guidelines') are systematically developed statements to assist practitioner and patient decisions about appropriate health care for specific clinical circumstances. ${ }^{1}$ The Scottish Dental Clinical Effectiveness Programme (SCDEP) http:// www.sdcep.org.uk was established in 2004 to provide guidance for the dental profession. For each clinical guideline, a guideline development group (GDG) assesses the best available evidence and reaches consensus to provide clinical recommendations. Their most recent guidance document relates to the prevention and treatment of periodontal diseases (primarily chronic periodontitis) in a primary dental care setting.

This guidance document has several components. The majority of the document is like a textbook in that it provides clear, stepby-step strategies for assessment and diagnosis - both clinical and radiographic, changing patient behavior (ie oral hygiene, smoking cessation), non-surgical therapy, maintenance of periodontal and implant health, policies for referral to specialty care and record keeping. It is designed to be used by general dentists, hygienists and therapists and would be particularly useful for education of dental clinicians. It is thorough and thoughtfully prepared. The assessment section includes recommendations for screening using the Basic Periodontal Examination (BPE - also known in North America as Periodontal Screening and Recording), when it is appropriate to take radiographs and which type to take, and a useful algorithm for assigning risk levels for development of periodontal diseases.

For development of the guidance document, the GDG formulated 20 key clinical questions. A systematic search of seven databases was conducted to identify the best available research up to October 2013. While periodontics is one area in oral health in which a significant amount of primary research and systematic reviews have been conducted, the evidence is not always strong, and the systematic reviews not always rigorously carried out. Nonetheless, the GDG evaluated the available evidence and developed clinical recommendations for each of the questions. When authoritative evidence was not available, the GDG made recommendations based on current best practice (presumably based on advice from experts). These recommendations are clearly identified in the 'Evidence into Practice' section. (Of course, there is also the ubiquitous 'Recommendations for Future Research' found in any guideline which, it seems, no researcher ever actually follows).

\section{Practice points}

While there are 20 key recommendations for the assessment and treatment of periodontal diseases in primary care, these have been summarised below.

In patients diagnosed with chronic periodontitis

- The most important factor for success is the patient's own plaque control, and that this is best achieved through engaging the patient and applying principles of behavior modification (TIPPS)

- Daily, effective plaque removal using a manual or rechargeable oscillating toothbrush and floss and/or interdental brush is recommended to maintain periodontal and peri-implant health.

In sites around teeth and implants demonstrating inflammation (ie probing depths $\geq 4 \mathrm{~mm}$ with sub-gingival deposits and/or which bleed on probing)

- Supra-gingival debridement and sub-gingival root surface instrumentation (using hand or power driven instruments; in one or more sessions) is the most appropriate treatment

- The addition of chemotherapeutics (local or systemic antimicrobials or host modulating therapies) provides no additional clinical benefit to mechanical debridement.

Following active therapy

- The length between recall appointments and the need for referral for specialty care should be individualised for each patient by assessing their periodontal status and risk for ongoing attachment loss at each recall visit.

One of the key messages in the guidance document is that management of periodontal health is a partnership between patient and clinician and requires a life-long commitment. Engaging patients in decision-making and therapy - through behavioural modification - is the key to success. To that end, a video of the implementation of TIPPS (Teach, Instruct, Practise, Plan and Support) is available from their website. Other tools for implementation include advice for medical practitioners and patient leaflets, including one for people with diabetes.

The potential benefit of any guideline is only as good as the quality of the guideline itself. This guidance document meets the requirements set out by the AGREE document ${ }^{2}$ in that the appropriate stakeholders were involved in its development, there are clear links between the evidence and the recommendations, the scope of the guideline and key recommendations are clear and specific, and additional tools are provided to facilitate application of the recommendations. Guidelines are not meant to replace clinical judgment. Nonetheless, this document provides excellent support for decision making between clinicians and their patients.

\section{Debora C. Matthews \\ Faculty of Dentistry, Dalhousie University Halifax, Nova Scotia, Canada}

1. Woolf $\mathrm{SH}$, Grol R, Hutchinson A, Eccles M, Grimshaw I. Clinical guidelines: potential benefits, limitations, and harms of clinical guidelines. Br Med / 1999; 318: 527-530.

2. AGREE Next Steps Consortium (2009). The AGREE II Instrument [Electronic version]. http://www.agreetrust.org [Accessed 15th August 2014]

Evidence-Based Dentistry (2014) 15, 68-69. doi:10.1038/sj.ebd.6401036 